\title{
Monitoring of ageing of the DELPHI Barrel RICH MWPC's
}

\author{
D. Toet et al
}

\begin{abstract}
We describe a method to monitor ageing effects in the Multiwire Proportional Chambers (MWPC's) of the Barrel Ring Imaging Cherenkov (BRICH) Detector of DELPHI. It is based on a measurement of plateau-curve characteristics and the shift of these as a function of the total collected charge. Results indicate that as yet no ageing effects are present.
\end{abstract}

Contribution to RICH'95, International Workshop on RICH Counters, Uppsala 12-16 June 1995 


$$
\text { , }
$$




\title{
Monitoring of ageing of the DELPHI Barrel RICH MWPC's
}

\author{
G. van Apeldoorn ${ }^{a}$, C. Bourdarios ${ }^{b}$, A. Buys ${ }^{b}$, M. Dracos ${ }^{c}$, P. Juillot ${ }^{c}$, N. Kjaer ${ }^{\text {d }}$, D. Z. Toet ${ }^{a *}$ \\ a NIKHEF-H, Postbus 41882, NL-1009 DB Amsterdam, The Netherlands

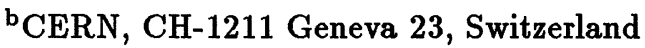

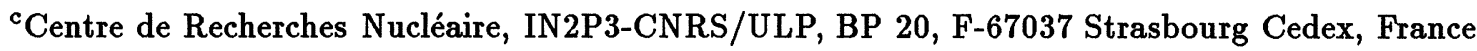 \\ ${ }^{\mathrm{d}}$ Niels Bohr Institute, Blegdamsvej 17, DK-2100 Copenhagen, Denmark
}

We describe a method to monitor ageing effects in the Multiwire Proportional Chambers (MWPC's) of the Barrel Ring Imaging Cherenkov (BRICH) Detector of DELPHI. It is based on a measurement of plateau-curve characteristics and the shift of these as a function of the total collected charge. Results indicate that as yet no ageing effects are present.

\section{INTRODUCTION}

In this paper we describe a method to monitor ageing of the wires of Multiwire Proportional Chambers (MWPC's). Ageing is the phenomenon that the efficiency of the MWPC for detection of electrons decreases as a function of the accumulated charge. Therefore ageing has to be monitored regularly.

We applied this method to the MWPC's of the Barrel Ring Imaging Cherenkov (BRICH) detector of the DELPHI experiment $[1,2]$ at LEP.

Our RICH detectors are equipped with liquid and gaseous radiators in which charged particles may give rise to Cherenkov radiation. This radiation is projected into quartz tubes filled with a photosensitive gas. The liberated photoelectrons drift in the electric field towards MWPC's [3], which equip each of the 48 tubes. The wire chambers can detect single photoelectrons created by the Cherenkov light, but they receive as well the much larger amount of ionisation electrons, $\approx 60 / \mathrm{cm}$, created by charged particles. The reported measurements concern the MWPC's of the BRICH.

Wire ageing has been the subject of many investigations [4], studying the phenomenon as a function of several parameters. Ageing effects increase with the amount of accumulated charge on the electrodes where the gas amplification or gas gain process takes place. The total accumu-

\footnotetext{
*Corresponding author
}

lated charge per unit (wire-) length is therefore the main parameter for the ageing process. Under an electron microscope aged wires are found to be coated by chemical deposits; analysis generally shows a strong presence of $\mathrm{C}, \mathrm{N}$ and $\mathrm{O}$ and some traces of $\mathrm{Si}$ [4]. These deposits are created in the avalanches from the different molecules present in the chamber gas, including impurities. They are responsible for the loss in gas gain. It has been shown that the presence of TMAE (see next section) in the driftgas accelerates ageing.

The effect of ageing is most directly observed as a shift to lower values of the pulse height distribution or as a decrease of anode current. We will however try to infer ageing from the analysis of the plateau-curve, as described in Section 3.

\section{THE BARREL RICH MWPC's AND THE DRIFT GAS}

The Barrel RICH MWPC's are described in [3]. We list here the main characteristics and the materials used in their construction (Fig.1):

- The chambers have a gain factor of $2 \times 10^{5}$ at the working point, i.e. at a cathode voltage of $-1450 \mathrm{~V}$ [5] in order to detect single photoelectrons efficiently.

- The anode wires are $20 \mu \mathrm{m}$ diameter goldplated tungsten wires, the pitch is $2.62 \mathrm{~mm}$ and they are positioned at $0.5 \mathrm{~mm}$ from the flat cathode plane. 


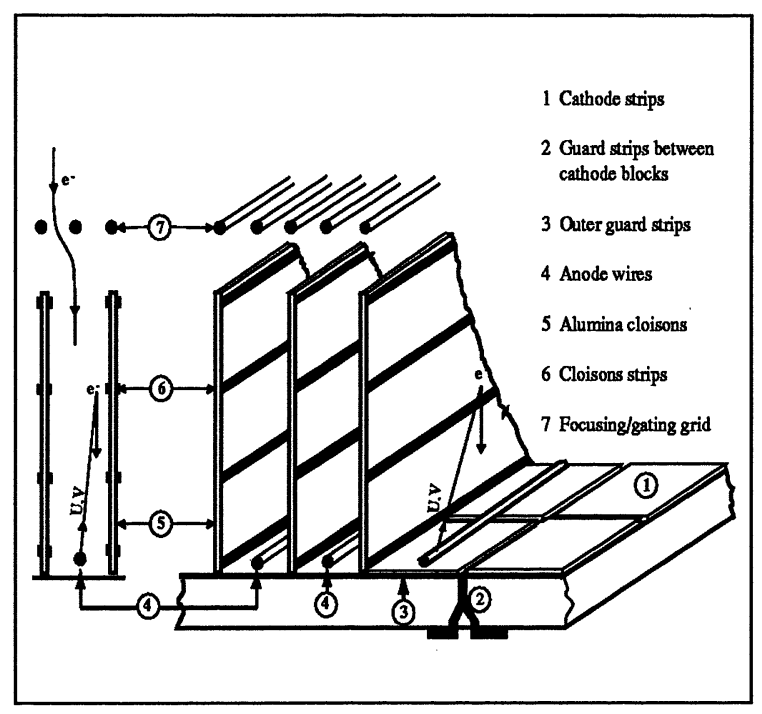

Figure 1. Schematic layout of the Barrel RICH MWPC's

- The cathode plane is divided into $3.8 \mathrm{~mm}$ wide/4 mm pitch gold plated copper strips on a G10 printed circuit board.

- Blinds between the wires act as an optical shielding against secondary (feedback) photons created in the avalanches. They consist of $9.8 \mathrm{~mm}$ high, $0.254 \mathrm{~mm}$ thick alumina sheets with potential strips on each side to focus the drifting electrons onto the anodes.

We did not take special precautions against ageing in the choice of our materials. The main material used for the chamber body, wire supports etc. is glass fibre-epoxy.

The chamber gas is a 3:1 mixture of methane and ethane. It is fed through a bubbler filled with TMAE (Tetrakis diMethyl Amine Ethylene) [6] at $28^{\circ} \mathrm{C}$. In this way the gas is doped with photo-sensitive TMAE vapour at the saturation pressure of 0.8 mbar.

\section{DESCRIPTION OF THE METHOD}

Measurements of the shift of the pulse height distribution as a function of the accumulated charge have been performed on a prototype chamber in a test beam [7]. Our conclusion was that the gas amplification at the working point would decrease to $50 \%$ of that of a new chamber not before having accumulated $2.5 \times 10^{-4} \mathrm{C} / \mathrm{cm}$.

Our readout system is not equipped to measure pulse heights. We therefore base our determination of ageing on the measurement of the plateau curves (count rate versus cathode voltage, Fig.2) after each year's running period.

Ageing will show up qualitatively as a change of the characteristics of this plateau curve, notably in a shift of the voltage at which the chamber starts to count and that where the plateau starts. For these measurements we carefully maintained the same conditions as for normal running.

The calibration system [2] was used to produce single photoelectrons in the drift tubes. Most tubes are equipped with a fibre-optics system in order to inject light into the tube at $\mathbf{4 5}$ accurately known places. These systems are fed by a common external UV lamp. Per pulse of the lamp an average of 0.1 photoelectron is created per fibre.

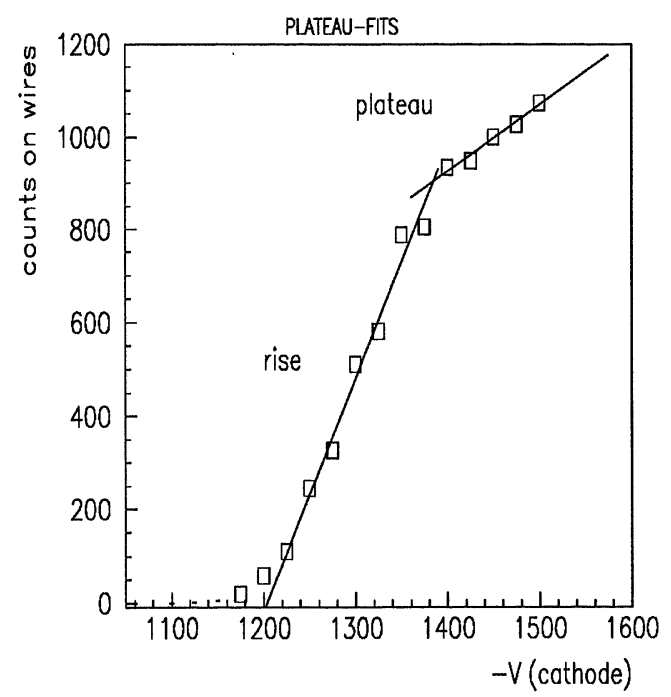

Figure 2. Example of a plateau curve and the lines fitted to it.

We group the chambers into groups of equal age in order to compare the plateau characteristics as a function of chamber lifetime. The chambers on 


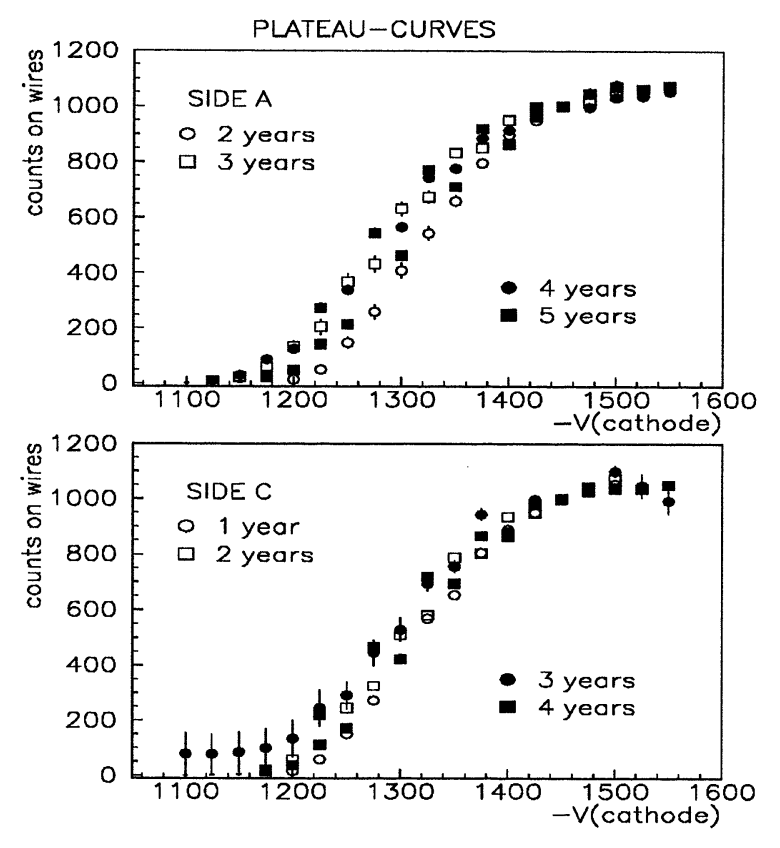

Figure 3. Plateau curves

one side (side A) of the BRICH were operational one run-year earlier than those on the opposite side (side C). They appear as such in the table and in the figures. The two sides are symmetrically located w.r.t. the interaction point.

The plateau curve was measured by varying the cathode voltage between $-1100 \mathrm{~V}$ and $-1550 \mathrm{~V}$ in steps of $25 \mathrm{~V}$. Voltages on the blinds, the gating wires, the transfer electrode and the drift field were varied accordingly. At each voltage we record the number of wire hits in each of the chambers separately.

In order to monitor and correct for any variations with time of the light yield, two chambers at each side (A and C) are kept at constant (working point) voltages. Since the calibration system does not distribute the same amount of light to each drift tube and this light distribution is likely to change from year to year, e.g. due to lamp replacement and/or positioning, we normalize the counting rate of each chamber to give the same value for all chambers $(1000$ hits $)$ at $-1450 \mathrm{~V}$.

Finally we obtain for each age group at each voltage the average number of normalized hits.
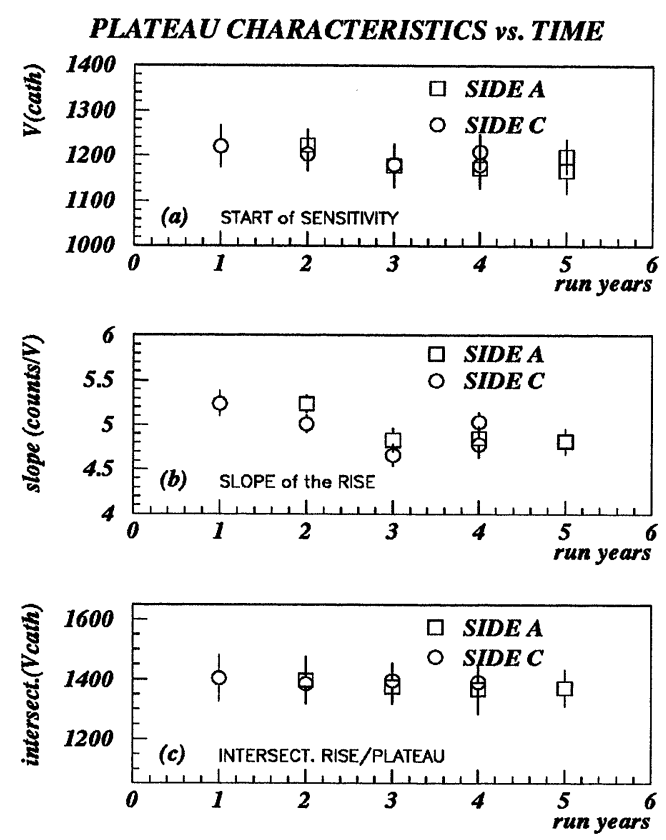

Figure 4. Plateau characteristics vs. number of run-years.

These points constitute the plateau-curve. We fit straight lines to the 'rise'- and to the 'plateau'part of this curve (see Fig.2).

The chambers of each age group did not undergo any intervention (remained in situ) during the 'lifetime' considered.

\section{RESULTS}

The plateau curves measured after the different running periods are shown in Fig.3.

The results of straight line fits to the rise of the curve and to the plateau part of it are given in Table 1 and are plotted in Fig. 4

As the measurements for 1994 were interrupted by a magnet ramp-down we give the results of the fits to the curves after and before the ramp-down separately ((1) and (2) in the Table).

Our method has a sensitivity to systematic shifts in voltage and counting efficiency of better than $40 \mathrm{~V}$ and $20 \%$ at $1350 \mathrm{~V}$ respectively. We see no deterioration - in fact a slight improvement - in the results over the years, now having collected some $3.5 \times 10^{7}$ incident electrons $/ \mathrm{cm}$ 


\begin{tabular}{|c|c|c|c|}
\hline 'age' & $\begin{array}{c}\text { gain starting point } \\
\text { (V) }\end{array}$ & $\begin{array}{c}\text { slope of rise } \\
\text { (hits/V) }\end{array}$ & $\begin{array}{l}\text { intersect. with } \\
\text { plateau line(V) }\end{array}$ \\
\hline $\begin{array}{c}1 \text { year } \\
\text { side C '91 }\end{array}$ & $1222 \pm 47$ & $5.24 \pm 0.14$ & $1403 \pm 78$ \\
\hline $\begin{array}{c}2 \text { years } \\
\text { side } A \text { '91 } \\
\text { side } C \text { '92 }\end{array}$ & $\begin{array}{l}1222 \pm 35 \\
1204 \pm 36\end{array}$ & $\begin{array}{l}5.24 \pm 0.10 \\
5.01 \pm 0.10\end{array}$ & $\begin{array}{l}1397 \pm 80 \\
1385 \pm 61\end{array}$ \\
\hline $\begin{array}{c}\text { years } \\
\text { side } A \text { '92 } \\
\text { side } C \text { '93 }\end{array}$ & $\begin{array}{l}1179 \pm 47 \\
1181 \pm 46\end{array}$ & $\begin{array}{l}4.82 \pm 0.13 \\
4.66 \pm 0.12 \\
\end{array}$ & $\begin{array}{l}1375 \pm 59 \\
1396 \pm 58\end{array}$ \\
\hline $\begin{array}{c}4 \text { years } \\
\text { side A '93 } \\
\text { side C '94 (1) } \\
\text { side C'94 (2) }\end{array}$ & $\begin{array}{l}1173 \pm 46 \\
1180 \pm 51 \\
1209 \pm 38\end{array}$ & $\begin{array}{l}4.85 \pm 0.13 \\
4.78 \pm 0.14 \\
5.03 \pm 0.11\end{array}$ & $\begin{array}{c}1366 \pm 81 \\
1390 \pm 57 \\
-\end{array}$ \\
\hline $\begin{array}{c}5 \text { years } \\
\text { side A '94 (1) } \\
\text { side A '94 (2) }\end{array}$ & $\begin{array}{l}1168 \pm 51 \\
1199 \pm 37\end{array}$ & $\begin{array}{l}4.81 \pm 0.14 \\
4.82 \pm 0.10\end{array}$ & $\begin{array}{c}1371 \pm 62 \\
-\end{array}$ \\
\hline
\end{tabular}

Table 1

Characteristics of plateau curves measured after different periods of ageing.

corresponding to $1.1 \times 10^{-6} \mathrm{C} / \mathrm{cm}$. We conclude from this that no ageing effects are visible.

\section{CONCLUSIONS}

We have described and presented the results of a method to qualitatively monitor the effects of ageing in our MWPC's on the counting efficiency. The results we measured confirm our expectations that ageing should not be measurable at the present level of integrated charge collected on the wires. We will continue to monitor for any future ageing effects with this method.

\section{REFERENCES}

1 P.Aarnio et al., Nucl. Instr. and Meth. $\mathbf{A 3 0 3}$ (1991) 233 and references therein.

2 E.G.Anassontzis et al., Nucl. Instr. and Meth. A323 (1992) 351 and references therein.

3 D.Bloch et al., Nucl. Instr. and Meth. A273 (1988) 847.

G. van Apeldoorn et al., Nucl. Instr. and Meth. A283 (1989) 767.
4 J.Va'vra, Nucl. Instr. and Meth. A252 (1986) 547.

J.Va'vra, IEEE Trans. Nucl. Sci. NS-34 (1987) 486. 547.

J.Va'vra, CRID MEMO nr.36 (1987).

J.Kadyk, Nucl. Instr. and Meth. A300 (1991) 436.

K.Martens et al., Nucl. Instr. and Meth. A343 (1994) 258.

M.Capeáns et al., Nucl. Instr. and Meth. A337 (1993) 122.

A.Algeri et al., Nucl. Instr. and Meth. A338 (1994) 348.

R.Bouclier et al., Nucl. Instr. and Meth. A346 (1994) 114.

5 M.Dracos and D.Loukas, Nucl. Instr. and Meth. A302 (1991) 241.

6 R.A.Holroyd et al., Nucl. Instr. and Meth. A261 (1987) 440.

7 D.Langerveld, D.Toet, W.Dulinski, Aging due to TMAE in a MWPC, Internal Report (unpublished) (1987). 